\title{
ACAULON FONTIQUERIANUM (POTTIACEAE), A NEW SPECIES TO THE BRYOPHYTE FLORA OF TURKEY AND SW ASIA
}

\author{
Mesut KIRMACI ${ }^{1} \&$ AdnAN ERdaĞ
}

\begin{abstract}
Acaulon fontiquerianum Casas \& Sérgio (Pottiaceae) is reported for the first time from Turkey and Southwest Asia. The species is briefly described and illustrated, and a key for Turkish Acaulon Müll. Hal. species is provided. The distribution of all Acaulon species known from Turkey is presented.
\end{abstract}

Key words: Acaulon, mosses, phytogeography, Turkey

Mesut Kırmacı \& Adnan Erdağ, Adnan Menderes Üniversitesi, Fen Edebiyat Fakültesi, Biyoloji Bölümü, 09100 Kepez-Aydın, Turkey; e-mail: mkirmaci@adu.edu.tr\&aerdag@adu.edu.tr

\section{INTRODUCTION}

Pottiaceae is the richest family in the bryoflora of Turkey (Kürschner \& Frey 2011), due to its various adaptations to the prevailing dry summer climate, with its long arid season, and the presence of many sites offering suitable habitats for these species of harsh environments.

Within the family, the genus Acaulon Müll. Hal. is represented by three species in Turkey; eight species are distributed across the Mediterranean area, including the Balearic Islands, Crete, Croatia, Israel, Montenegro, Morocco, Portugal, Sardinia, Sicily and Spain, and the Canary Islands in the Atlantic Ocean (Ros et al. 2013). They include A. casasianum Brugués \& H. A. Crum, A. dertosense Casas, Sérgio, Cros \& Brugués, $A$. fontiquerianum Casas \& Sérgio, A. longifolium Herrnst. \& Heyn, A. mediterraneum Limpr., A. muticum (Hedw.) Müll. Hal., A piligerum (De Not.) Limpr. and $A$. triquetrum (Spruce) Müll. Hal.

In Turkey, A. mediterraneum, A. muticum and $A$. triquetrum were recorded previously (Kürschner \& Erdağ 2005; Ros et al. 2013). Acaulon fontiquerianum was relatively recently described by Casas and Sergio (1990) from southern Spain, Portugal, France and Corsica (Sérgio et al. 1993),

\footnotetext{
1 Corresponding author
}

and more recently reported from the Canary Islands (Dirkse et al. 1993), Sicíly (Lo Giudice 1995) and Sardinia (Cogoni \& Scrugli 2000). The new record from Turkey has a collection history similar to that of Didymodon bistratosus Hébr. \& R. B. Pierrot, which was discovered by Hébrard and Pierrot (1994) on the Iberian Peninsula and recorded eleven years later in Turkey (Erdağ \& Kürschner 2005); minute and often ephemeral bryophyte species can easily be overlooked in field studies.

\section{RESULTS}

Acaulon fontiquerianum Casas \& Sérgio

Figs $1 \& 2$

Cryptog. Bryol. Lichénol. 11: 61. 1990.

Small, greenish (becoming brownish with age) ephemeral plants in loose turfs and scattered among other species, up to $3 \mathrm{~mm}$ high, with erect stems sometimes branched at base; rhizoids as long as stems; leaves 8-10, larger in upper parts, narrower in sterile individuals, concave, broadly ovate, $c a 1.25 \mathrm{~mm}$ long, erect when moist, apices acute and incurved when dry; leaf margins plane, entire and weakly recurved below; lamina 


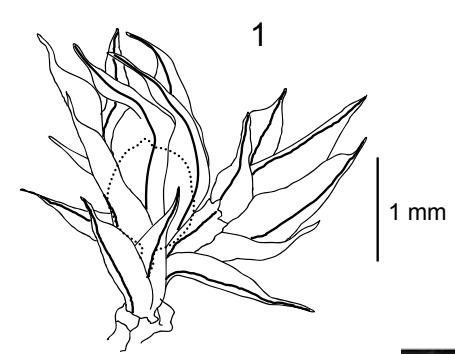

3

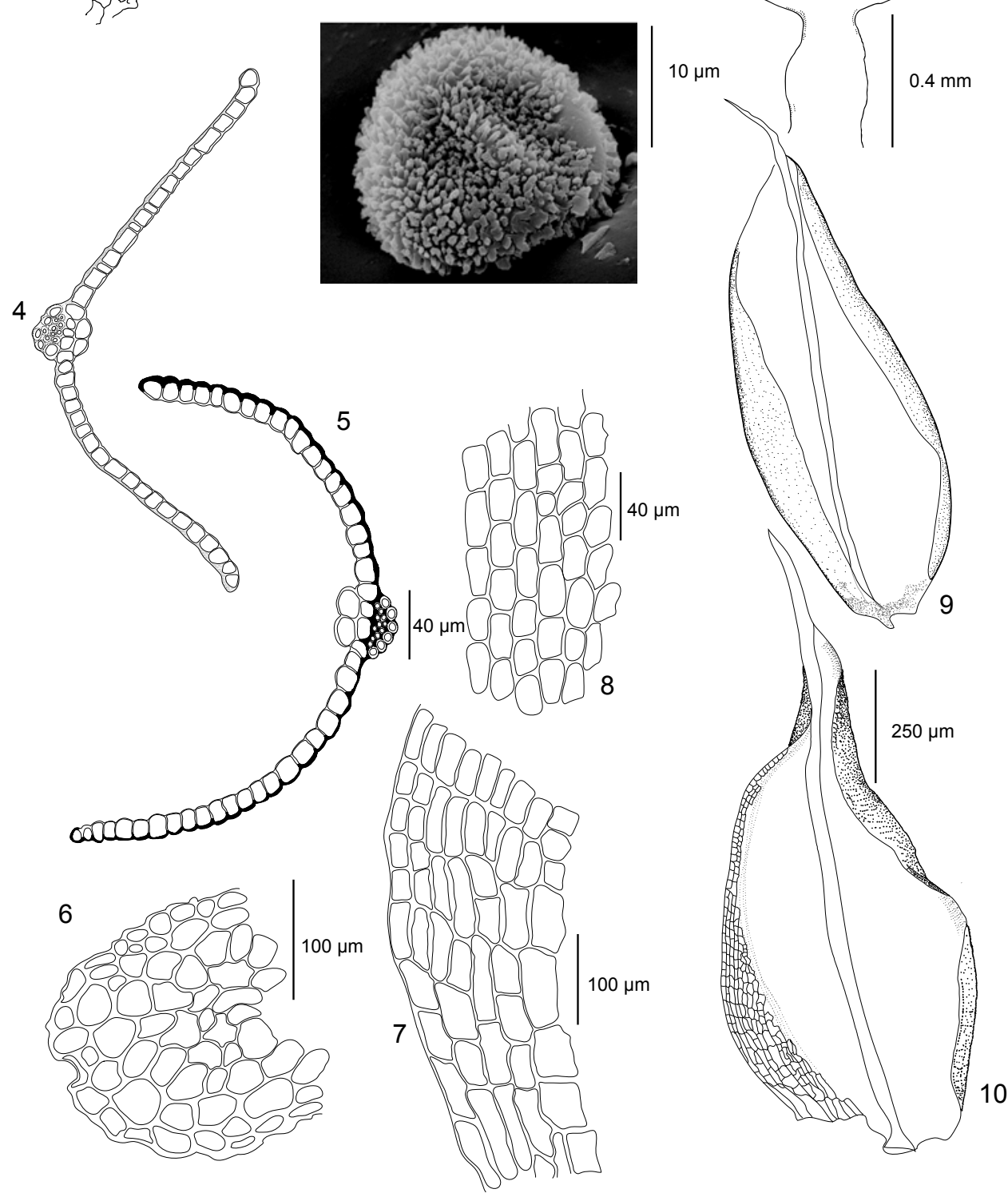

Fig. 1. Acaulon fontiquerianum Casas \& Sérgio: 1 - habit, 2 - capsule, 3 - spore (SEM), 4 - cross section of lower part of leaf, 5 - cross section of middle part of leaf, 6 - cross section of stem, 7 - basal cells, 8 - midleaf cells, $9 \& 10$ - leaves (all from AYDN 3401). 
unistratose throughout; costa longly excurrent (up to $250 \mu \mathrm{m}), 42 \mu \mathrm{m}$ wide at base, rounded in cross section; dorsal stereid band 3-5 cell thick, with 2 or more prominent cells on ventral side; median and upper laminal cells subquadrate, smooth, (12-)17-22(-30) $\mu \mathrm{m}$, basal cells rectangular, up to $75 \mu \mathrm{m}$ long towards base, ca $35 \mu \mathrm{m}$ long, in upper parts 12-20 $\mu \mathrm{m}$ wide; capsule immersed, subglobose, cleistocarpous, $\mathrm{ca}$ 0.7(0.8) $\mathrm{mm}$ in diameter; seta erect, ca $0.4 \mathrm{~mm}$ long; lid conical; calyptra small; spores papillose, $22-25 \mu \mathrm{m}$ in diameter.

Specimen examined: TURKEY. Prov. Denizli, Nikfer county, Bozdağ, Yorga Plateau, Karaçukur, alt. $2100 \mathrm{~m}, 37^{\circ} 17^{\prime} 49.93^{\prime \prime} \mathrm{N} ; 29^{\circ} 10^{\prime} 01.26^{\prime \prime} \mathrm{E}$, on soil of meadow, 9 June 2012, M. Kırmacı (AYDN 3401).

Acaulon fontiquerianum was collected in W Turkey, Bozdağ (Denizli) by the junior author in 2012. Bozdağ Mountain (2421 m a.s.1.) is situated at the westernmost extensions of the Taurus range. Here, A. fontiquerianum was collected among grasses of a mountain meadow on calcareous soil at $2100 \mathrm{~m}$ a.s.l. This site reflects the large altitudinal distribution of the species, which previously was recorded from sea level (cf. Cogoni \& Scrugli 2000; Casas et al. 1990; Sérgio et al. 1993). Associates at the Turkish site are Fossombronia sp., Barbula convoluta Hedw.,
Bryum dichotomum Hedw., Dicranella howei Renauld \& Cardot and Tortula cuneifolia (Dicks.) Turner. These interesting bryophyte communities are still poorly known. Only one study on ephemeral bryophyte communities of Turkey is available (Kürschner et al. 2007).

\section{Key to the TURKISH ACAULON SPECIES \\ (after Kürschner \& Frey 2011, modified)}

1. Plants triangular when viewed from above; leaves strongly keeled; costa long excurrent; seta arcuate . . .......... A. triquetrum (Spruce) Müll. Hal.

1. Plants rounded when viewed from above; leaves not keeled; costa excurrent or ending in apex; seta straight; capsule erect . . . . . . . . . . . . 2

2. Leaves broadly ovate, concave; leaf margins plane or weakly recurved; median leaf cells $1.0-1.5$ times as long as wide; costa excurrent in a long apiculus to $250 \mu \mathrm{m}$ long; spores $22-25 \mu \mathrm{m}$ in diameter ..... A. fontiquerianum Casas \& Sérgio

2 . Leaves broad-ovate to elliptic, concave, toothed at apex; leaf margins recurved; median leaf cells mostly 2-3 times as long as wide; costa ending below apex to shortly excurrent; spores $30-50 \mu \mathrm{m}$ in diameter ................... 3

3. Leaves toothed at apex; spores papillose to finely verrucose ..... A. muticum (Hedw.) Müll. Hal. 3. Leaves entire; spores spinulose . . . . . . . . . . . ............... mediterraneum Limpr.

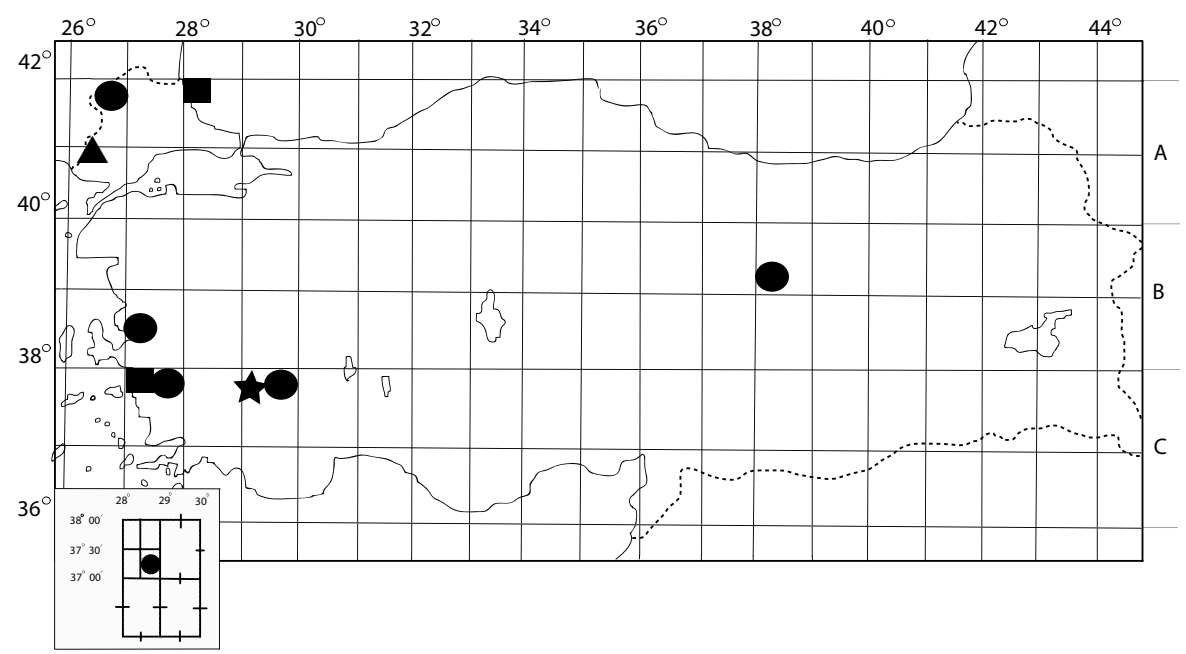

Fig. 2. Distribution of Acaulon species in Turkey. $\star-A$. fontiquerianum Casas \& Sérgio,

- - A. muticum (Hedw.) Müll. Hal., - A. triquetrum (Spruce) Müll. Hal. 


\section{Distribution OF ACAULON SPECIES IN TURKEY}

Acaulon mediterraneum Limpr.

Fig. 2

Laubm. Deutschl. 1: 180. 1885.

Prov. Edirne: W Hamidiye, $41^{\circ} 09^{\prime} 27^{\prime \prime} \mathrm{N}$, $26^{\circ} 38^{\prime} 57^{\prime \prime}$, alt. $109 \mathrm{~m}$, on calcareous soil, 21-27 April 2006, R. Natcheva, M. Coşkun \& A. Cayır (Natcheva et al. 2008).

Acaulon muticum (Hedw.) Müll. Hal. Fig. 2

Bot. Zeitung (Berlin) 5: 99. 1847. - Phascum muticum Hedwig, Sp. Musc. Frond.: 23. 1801. - Acaulon minus (Hook. \& Taylor) A. Jaeger, Ber. St. Gall. Naturw. Ges. 1868-69: 78. 1869. - Acaulon muticum var. minus (Hook. \& Taylor) Bruch \& Schimp., Bryol. Eur. 1: 17. 1849.

Prov. Erzincan: Kemaliye, Yeşilyamaç köyü, $39^{\circ} 15^{\prime} 41.5^{\prime \prime} \mathrm{N} 38^{\circ} 33^{\prime} 08^{\prime \prime} \mathrm{E}$, alt. $1420 \mathrm{~m}, 12$ April 2006, A. Erdağ 06/14 (AYDN 3402). - Prov. İzmiR: Yamanlar Dağı, Karagöl Dere, alt. 815 m, muddy soil bank, 12 July 1967, K. Walther 6103 (Walther 1970). - Prov. Aydin: İmamköy, $37^{\circ} 52^{\prime} 5.9^{\prime \prime} \mathrm{N}, 27^{\circ} 54^{\prime} 27.9^{\prime \prime} \mathrm{E}$, alt. $150 \mathrm{~m}$, on soil, 2 July 2008, E. Agcagil (Kirmac1 \& Agcagil 2009).

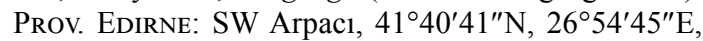
156 m, dry grassland, 21-27 April 2006, R. Natcheva, M. Coskun \& A. Cayir (Natcheva et al. 2008). - Prov.

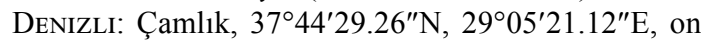
soil, alt. $500 \mathrm{~m}$, and alt. $450 \mathrm{~m}$., Pinus brutia forest, 30 May 2006, M. Kırmacı (Kırmacı \& Erdağ 2010).

Acaulon triquetrum (Spruce) Müll. Hal. Fig. 2 Bot. Zeitung (Berlin) 5: 100. 1847. - Phascum triquetrum Spruce, London J. Bot. 4: 189. 1845.

Prov. İzmir: SE Kuşadası, alt. $300 \mathrm{~m}$, on soil, 12 Juni 1965, K. Walther 2552d (Walther 1967). - Prov. ThraCE: Yıldız Dağları north of Kıyıköy, alt. 50 m, on soil, 13 May 2000, B. Papp \& M. Sabovljević (Papp \& Sabovljević 2003).

ACKNOWLEDGEMENTS. We are grateful to Emre Agcagil (MS student of the Adnan Menderes University) for his kind help during field studies, to Harald Kürschner (Berlin) for providing valuable information on the distribution of the Turkish species, and to the anonymous reviewers for helpful suggestions on the manuscript. The Scientific Research Project Unit of Adnan Menderes University provided financial support to Mesut Kırmac1 (FEF 11-006).

\section{REFERENCES}

Casas C. \& Sérgio C. 1990. Acaulon fontiquerianum sp. nov. de la Peninsula Ibérica. Cryptog. Bryol. Lichénol. 11: $57-61$.

Casas C., Sérgio C., Cros R. M. \& Brugués M. 1990. Datos sobre el género Acaulon en la Peninsula Ibérica. Cryptog. Bryol. Lichénol. 11: 63-70.

Cogoni A. \& Scrugli A. 2000. Acaulon fontiquerianum Casas et Sergio (Musci, Pottiaceae) new to Sardinia. Cryptog. Bryol. 21: 285-288.

Dirkse G. M,, Bouman A. C. \& Losada-Lima A. 1993. Bryophytes of the Canary Islands. An annotated checklist. Cryptog. Bryol. Lichénol. 14: 1-47.

ERDă̆ A. \& Kürschner H. 2005. Didymodon bistratosus Hébr. \& R. B. Pierrot (Pottiaceae, Bryopsida), a new record to the bryophyte flora of Turkey. Cryptog. Bryol. 26: 233-236.

HÉbrard J. P. \& Pierrot R. B. 1994. Didymodon bistratosus (Pottiaceae, Musci), espèce nouvelle du sud de l'Espagne (environs de Ronda, province Málaga). Nova Hedwigia 59: 353-364.

KIRMACI M. \& AGCAGIL E. 2009. The bryophyte flora in the urban area of Aydin (Turkey). International Journal of Botany 5(3): 216-225.

KIRMACI M. \& ERDAĞ A. 2010. The bryophyte flora of Babadağ (Denizli/Turkey). Biological Diversity and Conservation 3(2): 72-78.

KüRSCHNER H. \& ERDAĞ A. 2005. Bryophytes of Turkey: An annotated reference list with synonyms from the recent literature and an annotated list of Turkish bryological literature. Turkish Journal of Botany 29: 95-154.

KürSCHNER H. \& FREY W. 2011. Liverworts, mosses and hornworts of Southwest Asia (Marchantiophyta, Bryophyta, Anthocerotophyta). Nova Hedwigia Beih. 139: 1-240.

Kürschner H., Parolly G., Erdă̆ A. \& Eren Ö. 2007. Synanthropic bryophyte communities new to Western Turkey - syntaxonomy, synecology and life sndromes. Nova Hedwigia 84: 459-478.

Lo Giudice R. 1995. Acaulon fontiquerianum Casas \& Sérgio (Pottiaceae, Bryophytina), new to the bryoflora of Italy. Fl. Medit. 5: 69-72.

Natcheva R., CoşKun M. \& ÇAYIR A. 2008. Contribution to the bryophyte flora of European Turkey. Phytologia Balcan. 14(3): 335-341

Papp B. \& SABovluević M. 2003. Contribution to the bryophyte flora of Turkish Thrace. Studia Bot. Hung. 34: 43-54.

Ros R M., Mazimpaka V., Abou-Salama U., Aleffi M., Blockeel T. L., Brugués M., Cros R. M., Dia M. G., Dirkse G. M., Draper I., El SaAdawi W., Erda $\breve{\text { A., }}$ Ganeva A., Gabriel R. M. A., Gonzalez-Mancebo J. M., Granger C., Herrnstadt I., Hugonnot V., KhaLIL K., KÜRschner H., Losada-Lima A., Luis L., Mifsud 
S. D., Privitera M., Puglisi M., Sabovijević M., Sérgio C., Shabbara H., Sim-Sim M., Sotiaux A., Tacchi R., Vanderpoorten A. \& Werner O. 2013. Mosses of the Mediterranean, an annotated checklist. Cryptog. Bryol. 34: 99-283.

Sérgio C., Hébrard J. P. \& CASAs C. 1993. Acaulon fontiquerianum Casas et Sergio (Musci, Pottiaceae) nouveau pour la bryoflore du Portugal, de France et de Corse. Orsis 8: 11-19.

WALther K. 1967. Beiträge zur Moosflora Westanatoliens: I. Mitt. Staatsinst. Allg. Bot. Hamburg. 12: 129-186.

WALther K. 1970. Beiträge zur Moosflora Westanatoliens II. Mitt. Staatsinst. Allg. Bot. Hamburg. 13: 167-180.

Received 31 January 2014 